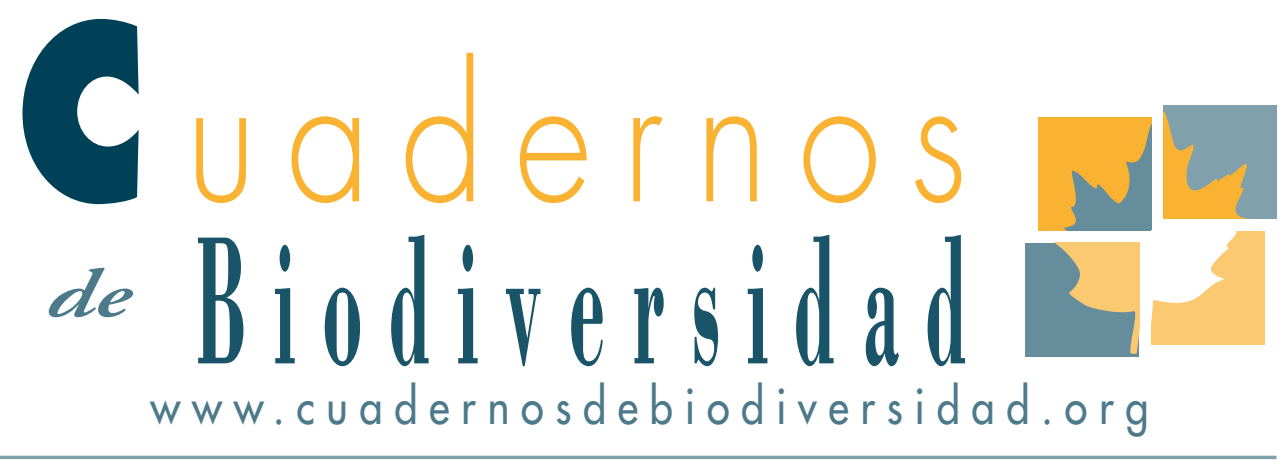

\title{
La bellota de Quercus insignis Martens \& Galeotti, 1843, la más grande del mundo
}

\section{Y. García-de la Cruz ${ }^{1 *}$, J. Becerra-Zalaveta ${ }^{2}$, P. A. Quintanar- Isaías ${ }^{3}$, J. M. Ramos-Prado ${ }^{4}$ y A.M. Hernández-Ramírez ${ }^{4}$}

i Centro de Investigaciones Tropicales, Universidad Veracruzana. Calle Araucarias s/n. Col 2 i de Marzo. Interior de la Ex-hacienda Lucas. Martín. C. P. 9ioi 9. Xalapa, Veracruz, MÉxico.

2 Instituto Tecnológico Superior de Perote. Km 2.5 Carretera Federal Perote-México, C.P. 9i 270. Perote, Veracruz, México.

2 Universidad Autónoma Metropolitana, Unidad Iztapalapa. Laboratorio de Tecnología de la Madera. Av. San Rafael Atlixco i86, Col. Vicentina, C.P. 09340. Delegación Iztapalapa, Distrito Federal, MÉxico.

3 Centro de Ecoalfabetización y Diálogo de Saberes, Av. de las Culturas Veracruzanas No. i, Col. Emiliano Zapata, C.P. 9io6o. Xalapa, Veracruz, México.

*AUTOR DE CORRESPONDENCIA: YURELII@HOTMAIL.COM

\section{ABSTRACT}

Quercus insignis M. Martens \& Galeotti, better known as "chicalaba" in Mexico is a critically endangered oak species and it is characterized by producing the biggest acorns in the world. Despite of its ecological importance as a dominant component in cloud forests in Veracruz, Mexico, its habitat is facing high deforestation rates. In this review, we describe the chicalaba, its distribution, uses and some ecological aspects to promote its propagation and management including ecological restoration programs.

\section{KEY WORDS:}

Quercus insignis, chicalaba, critical endangered oak species, biological description, current state of knowledge.

\section{INTRODUCCIÓN}

El término bellota (del árabe ballūta, encina; Real Academia Española, 2001) se refiere al fruto característico de las especies del género Quercus, 
de la familia Fagaceae. Botánicamente, la bellota se clasifica como una nuez, adherida a menudo a una cúpula o involucro. Algunas bellotas son comestibles y se venden en los mercados regionales del Mediterráneo y también en el Norte de México. Se conocen referencias de que los primeros habitantes californianos consumían estos frutos como alimento (Kroeber, 1925).

La forma de vida de los Quercus, denominados también encinos, es principalmente arbórea y arbustiva. Se distribuyen en regiones tropicales, subtropicales y templadas, en el Sureste de Asia, Noreste de África, la zona mediterránea de Europa, Norteamérica, Centroamérica y parte de Sudamérica (Valencia, 2004). En América, su distribución se restringe a elevaciones superiores a los $500 \mathrm{~m}$ hasta los $3500 \mathrm{~m}$, tales como las sierras de México y Cuba, las montañas de Belice, Guatemala y las tierras altas de Honduras y Nicaragua, las cordilleras Panameñas y los Andes Colombianos (Kapelle, 2006). Se tienen registros fósiles de encinos que se remontan al período Cretácico, pero su mayor diversificación ocurrió a principios del Oligoceno, es decir, hace unos 33 millones de años aproximadamente (Axelrod, 1983).

A nivel mundial se calcula que existen 500 especies de Quercus, de las cuales 160 se encuentran en México (109 endémicas) y 38 en el estado de Veracruz (Valencia, 2004; Oldfield \& Eastwood, 2007). Los encinos constituyen el principal tipo de plantas de los sistemas vegetales montañosos de México, es decir, de los bosques templados de encino, pinoencino, bosque de niebla y, ocasionalmente, matorrales y bosques tropicales caducifolios (Arizaga, 2009). De toda la riqueza de especies que presenta México, existe una especie muy peculiar cuya principal característica es el tamaño de su bellota, considerándose como la más grande del mundo.

\section{¿LA CONOCES?}

Comúnmente es conocida en Veracruz como "chicalaba", y científicamente como Quercus insignis. El ejemplar tipo, es decir, la primera muestra empleada para hacer la descripción de este taxón fue colectada en el Volcán Pico de Orizaba, Veracruz, por Galeotti (Núm. Colecta: 123, Año: 1840) y publicada en 1843 en el Bolletin de l'Académie Royale des Sciences et des Belles-Lettres de Bruxelles. Pertenece al subgénero Lepidobalanus (encino blanco) y está reconocida como una especie críticamente amenazada dentro de la lista roja de la UICN (Unión Internacional para la Conservación de la Naturaleza) ya que el tamaño de sus poblaciones se ha visto reducido por el cambio del uso del suelo y por la fragmentación su hábitat (González-Espinoza et al., 2011).

\section{DESCRIPCIÓN}

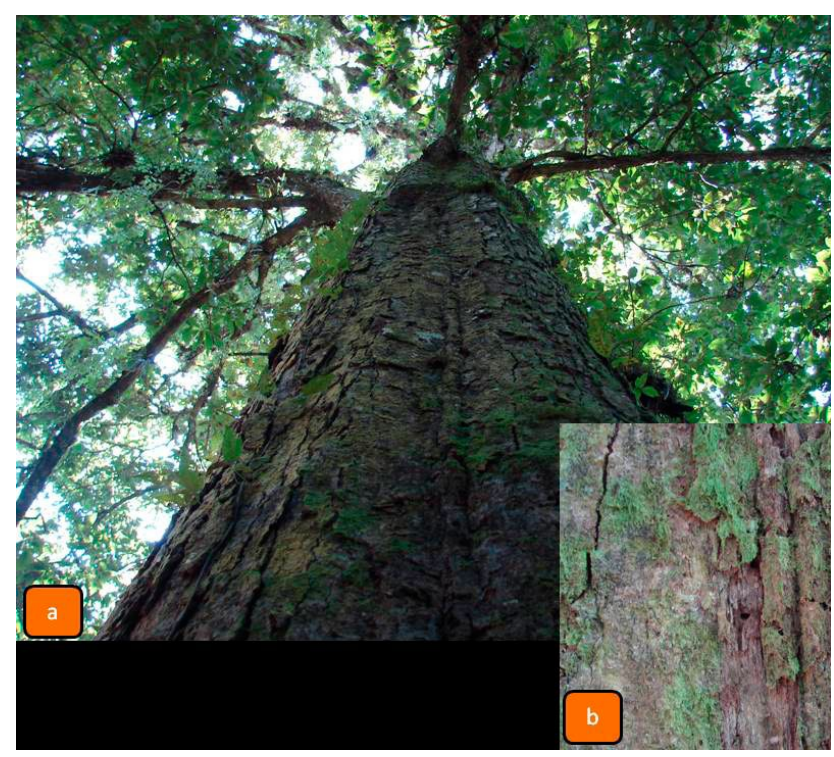

Fig. 1. a) Árbol de chicalaba, b) corteza

La chicalaba es un árbol que alcanza 30-40 metros de altura, tiene un tronco o fuste recto de hasta 1.3 $\mathrm{m}$ de diámetro y su corteza es grisácea y fisurada en forma de costillas paralelas donde habitan diversos líquenes y musgos (Figura 1). Su copa es notablemente redonda, sus hojas son en forma de elipse $\mathrm{u}$ obovadas y miden hasta $25 \mathrm{~cm}$ de largo y $4-9 \mathrm{~cm}$ de ancho, algo tomentosas (con pelos) cuando jóvenes y casi glabras (sin pelos) en la madurez. El margen o borde de sus hojas es entero u ondulado con 7-20 pares de pequeños dientes que se encuentran a partir de $2 / 3$ de la parte basal de la hoja. De acuerdo al tipo de nervadura, las hojas son penninervadas, es decir, 
los nervios secundarios laterales se originan en un solo nervio principal, los cuales están impresos en ambas caras de la hoja (Figura 2). Las plántulas de esta especie poseen tallos densamente tomentosos con tricomas amarillo-anaranjados, glabros en el tercer año, cuando la superficie se torna de gris a blanca (Flora de Nicaragua, 2009). Sus yemas son glabras, ovoides de 10 por $15 \mathrm{~mm}$ y sus estípulas son caedizas (Martínez, 1981).

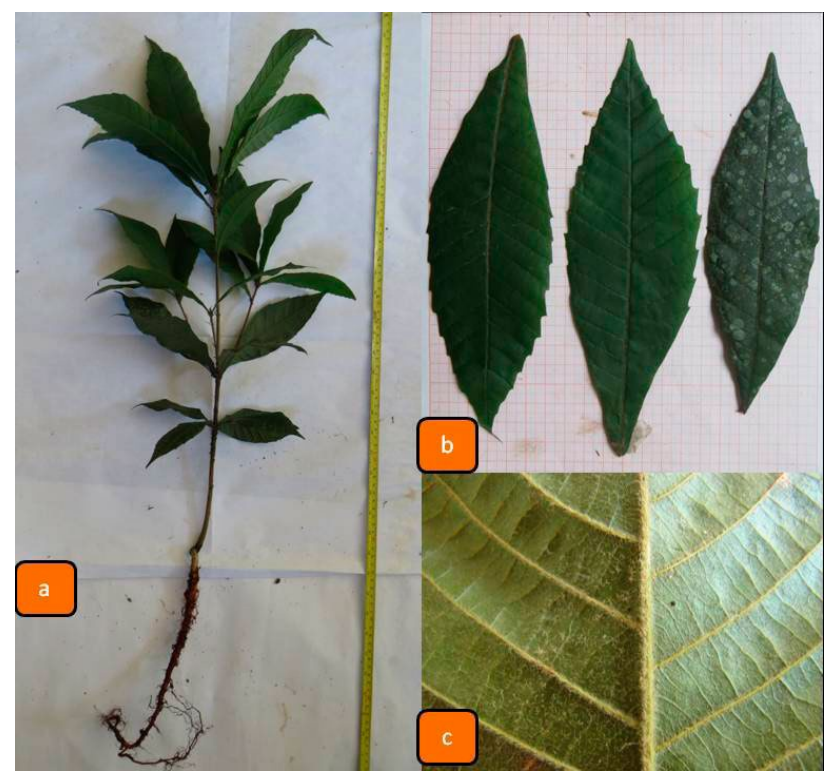

Fig. 2. a) Plántula de un año de edad, b) Hojas, c) Nervaduras

Es una especie monoica con flores unisexuales (existen inflorescencias femeninas y masculinas en el mismo árbol). Las inflorescencias masculinas miden 4-14 cm de largo, perianto (estructura floral que corresponde a la envoltura que rodea a los órganos sexuales) de 1.5-2 $\mathrm{mm}$ de largo, pubescente por dentro y por fuera, anteras glabras; mientras que las inflorescencias femeninas miden de $1-2 \mathrm{~cm}$ de largo, con 1-4 flores. Ambos tipos de flores son diminutas, colgantes y amarillentas. El transporte de polen entre las flores masculinas y femeninas es realizado por el viento (polinización anemófila). Las flores pueden permanecer hasta un mes en el árbol El período de floración inicia en marzo y fructifica de julio a noviembre (Benítez et al., 2004; Arizaga et al., 2009).
Las bellotas derivan de un ovario tricarpelar y cada carpelo posee dos o rara vez tres óvulos, de los cuales sólo uno se desarrolla, el resto aborta y permanecen conectados al integumento del óvulo funcional (Zavala, 1989). La chicalaba produce anualmente bellotas de gran tamaño, las cuales son anuales y solitarias, de color verde cuando están inmaduras y se tornan de color café una vez que maduran, llegando a medir 5,2 cm largo, 6,4 cm de ancho y pesar hasta $100 \mathrm{gr}$ (Figura 3). El esplendor de estos frutos hace alarde al término "insignis", en cuyo caso se refiere a algo destacado, importante, célebre o notable.

La forma del fruto es parecida a la de un trompo y sin duda, es el alimento preferido de las ardillas, venados, ratones y otros roedores. Los frutos están cubiertos en su base de unión con la rama por una estructura endurecida de forma cóncava llamada cúpula o involucro. Las cúpulas de la chicalaba tienen de 4-10 cm de ancho, son densamente tomentosas en el interior y poseen escamas gruesas en la parte externa de 6-10 mm de largo y 2-5 mm de ancho. La cúpula es una característica importante dentro del mundo de la taxonomía botánica, ya que se considera como un carácter diagnostico que define a la familia Fagaceae (Borgardt \& Pigg, 1999).

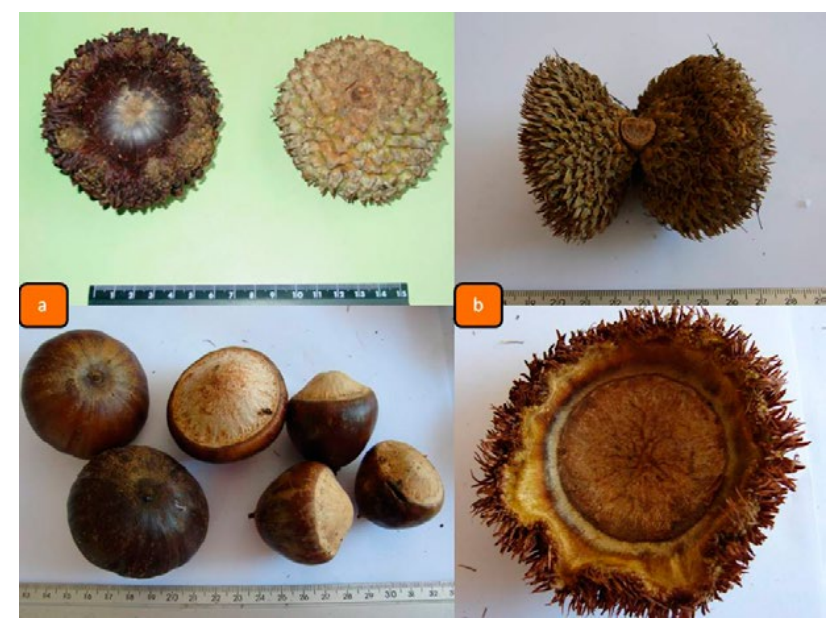

Fig. 3. a) Bellotas y b) cúpulas 


\section{DISTRIBUCIÓN}

Se distribuye en México y algunos países de Centroamérica (Guatemala, Belice, Honduras, Nicaragua, Costa Rica y Panamá). En México, se encuentra en los estados de Jalisco, Chiapas, Oaxaca, Guerrero y Veracruz (Zavala, 1989; Valencia, 1995). La chicalaba veracruzana se localiza en altitudes que van de los 900 a los 1600 msnm y habita en el bosque de niebla (bosque mesófilo de montańa) sobre suelos arcillosos, o en ocasiones se encuentra como parte de remanentes naturales asociados a los agroecosistemnas cafetaleros de la región (Benítez et al., 2004). En la zona centro del estado de Veracruz, esta especie se encuentra formando poblaciones naturales de baja densidad en los municipios de Huatusco, Zentla, Totutla y zonas adyacentes.

A pesar de esta amplia distribución, sus poblaciones no son muy densas, lo que aunado a los factores perturbadores la coloca en un estado de riesgo alto de conservación. Por este motivo, los estudios sobre su biología y ecología, nos permitirán comprender su distribución, su dinámica poblacional y características ecofisiológicas, indispensables para establecer las bases de programas viables de restauración ecológica.

\section{USOS}

En Veracruz se usa para la construcción y como materia prima para hacer hermosas artesanías, pero desafortunadamente también se aprovecha como leña y carbón (Oldfield \& Eastwood, 2007). En otros estados se ha reportado el uso de los frutos para alimentar a los cerdos (Benítez et al., 2004). Aunque no se tienen datos científicos sobre esta actividad, es interesante conocer si el contenido nutritivo de los frutos promueve el crecimiento del ganado porcino y mejora la calidad de su carne. Aunado a lo anterior, la venta de germoplasma (semillas) representa una alternativa ecológicamente viable y económicamente complementaria para los pobladores locales. Estos usos han mermado sus poblaciones de manera significativa. A pesar de ello, no existen planes de manejo para esta especie y mucho menos está incluida en la Norma Oficial Mexicana-059-SEMARNAT-2010.
Por lo que es evidente la necesidad de crear estrategias que fomenten la conservación de su hábitat y la propagación de esta emblemática especie para restaurar zonas perturbadas de bosque de niebla.

\section{DATOS ECOLÓGICOS}

A pesar de la importancia de esta especie en México y en el estado de Veracruz; existen pocos estudios sobre su biología y ecología. Quercus insignis coexiste con otras especies arbóreas del bosque de niebla como el ocozote (Liquidambar styraciflua), marangola (Clethra mexicana), varias especies de encinos como Quercus laurina, Q. leiophylla y $Q$. sartorii, cerillo (Myrsine coriacea), huevos de gato (Turpinia insignis), azahar de monte (Styrax glabrescens), entre otros (Williams-Linera, 2002; WilliamsLinera \& López, 2008). En condiciones de sombra intermedia, es decir, debajo de árboles y arbustos, la chicalaba llega a crecer de 80 a $100 \mathrm{~cm}$ (en dos años) y sobreviven el $34.4 \%$ de los individuos (MontesHernández \& López-Barrera, 2013). Su crecimiento llega a ser mayor cuando se encuentra en suelos (sustratos) orgánicos y modificados por la actividad de las lombrices de tierra (Pontoscolex corethrurus). Por ello, se ha recomendado la incorporación de suelos orgánicos en presencia de lombrices y otras plantas afines a la chicalaba, como medios de diversificación productiva dentro de los programas de reforestación asistida a nivel local y regional (Avendaño-Yañez et al., 2014).

\section{SI LA CHICALABA TIENE UNA AMPLIA DISTRIBUCIÓN ¿POR QUÉ ESTÁ EN RIESGO EN MÉXICO?}

Porque el tamaño de sus poblaciones se han reducido en más del $80 \%$ en los últimos 10 años. $\mathrm{Su}$ principal hábitat, el bosque de niebla, se ha fragmentado seriamente debido a la deforestación provocada por el crecimiento urbano, así como la creación de potreros y zonas agrícolas (WilliamsLinera, 2007). Junto con lo anterior, la distribución de esta especie en el bosque de niebla en el estado 
de Veracruz está limitada a algunos sitios de la zona centro y en la sierra de Los Tuxtlas donde la densidad de individuos es baja y al parecer presenta problemas de regeneración natural (Guevara et al., 2004; González-Espinosa et al., 2012).

Aunque no conocemos con detalle los factores que colocan a esta especie en estado de alto riesgo, lo anterior nos lleva a cuestionarnos: ¿Por qué sus poblaciones son aisladas y de baja densidad?, ¿Cómo se comportan la dinámica semillera en estas poblaciones? ¿Existen plagas o enfermedades que afecten la polinización y maduración del fruto?, ¿Qué papel tiene los factores microambientales en su dispersión y establecimiento?. Para ello, partimos de la siguiente pregunta:

\section{¿Qué se ha hecho y qué se está haciendo para conservar a la chicalaba?}

Las Unidades Productoras de Germoplasma Forestal, UPGF, son una estrategia de rescate, conservación, restauración y manejo forestal de los fragmentos de vegetación nativa de nuestro país (García-De la Cruz et al., 2011). En este contexto, la venta de germoplasma (semillas) de chicalaba representa una opción económicamente complementaria para los pobladores locales; ya que el valor comercial de un kilogramo de semillas (aproximadamente 17 bellotas) es de 40 pesos (aproximadamente), es decir, 2.96 dólares americanos o 2.31 euros; siendo una fuente económica adicional. Como muestra de ello, se encuentran algunos rodales semilleros identificados y documentados por la Comisión Nacional Forestal (CONAFOR) durante los años 90, en Huatusco, Zentla y Totutla.

El aprovechamiento de la semilla (bellota) está regulado por la NOM-007-SEMARNAT-1997, que establece los procedimientos, criterios y especificaciones para realizar el aprovechamiento, transporte y almacenamiento de ramas, hojas, flores, frutos y semillas. Para ello, los pobladores locales, generalmente dueños de los rodales semilleros, recolectan las semillas o nueces directamente del suelo, ya sin la cúpula, como señal de que la bellota está madura. Este germoplasma se almacena y transporta en costales de yute o malla para proveer ventilación y evitar la contaminación por hongos. Toda esta actividad, que va desde la floración hasta la recolección, está debidamente regulada dentro del rodal semillero, con el fin de no afectar la dinámica poblacional de la chicalaba en estos sitios. Además, la venta de bellotas está destinada a la producción de planta en vivero, la cual se dirige a los programas de reforestación $y /$ restauración fomentada por diversos programas de gobierno, Universidades y Asociaciones Civiles. Entre las más destacadas están la Comisión Nacional Forestal, PRONATURA y el Instituto de Ecología, A.C. Sin embargo, muchas veces no se da seguimiento a las plantaciones o no se publican los resultados de estos estudios.

Por otra parte, los registros que se tienen de la producción de planta de chicalaba dirigidos a los programas de reforestación del bosque de niebla incluyen: El vivero "Los Tanques" de la Secretaría de Desarrollo Forestal en Xalapa, "José Ángel Navar" de la CONAFOR Región X Golfo-Centro en Banderilla, "Las Cańadas" en Huatusco y el vivero de Plan de San Antonio en Coatepec.

Este último vivero, además de contar con un sistema tecnificado de producción de planta forestal, cuenta con un rodal semillero registrado por la CONAFOR, así como una microempresa de germoplasma que comercializa diversas semillas de encinos, entre las cuales figura la chicalaba. En este vivero se ha registrado hasta un $70 \%$ de germinación en un sustrato parecido al peat moss (turba) mezclado con tierra de monte, lo que permite mantener altos niveles de humedad para la germinación óptima de la bellota.

Actualmente, investigadores del Centro de Investigaciones Tropicales, el Centro de Ecoalfabetización y Diálogo de Saberes de la Universidad Veracruzana, el Instituto de Ecología A.C. y la Universidad Autónoma Metropolitana-Iztapalapa están llevando a cabo un proyecto de investigación sobre el establecimiento temprano de la chicalaba con el objetivo de proponer estrategias exitosas para la restauración del bosque de niebla en la zona centro de estado de Veracruz.

Es evidente que para tener éxito en los programas de restauración ecológica, es necesario crear protocolos y planes de manejo de la especie en cuestión, 
desde un enfoque holístico, es decir, que incluya una visión amplia de las especies que coexisten y sus interacciones, así como los factores abióticos que influyen en su supervivencia. Para ello, las acciones deben incluir estudios sobre:

- selección y registro de procedencia de los árboles madre de donde se obtiene el germoplasma,

- calidad de las semillas colectadas para determinar su viabilidad,

- germinación y crecimiento en vivero y campo, y bajo diferentes sustratos,

- registros de las condiciones abióticas donde crece (humedad relativa y temperatura del suelo así como del ambiente y cantidad de luz),

- análisis ecofisiológicos (conductividad hidráulica, estomática, tasa fotosintética y anatomía foliar),
- desempeño a través de plantaciones mixtas con especies nativas. Por ejemplo, si se ha registrado un crecimiento relativamente rápido en esta especie, podría servir para generar sombra en especies que lo requieren y tienen crecimiento más lento como el caso de otras especies de encino, o bien, a través del uso de especies que pertenecen a diferentes grupos funcionales

Actualmente, se tienen datos inéditos de germinación (50 al 70\%) bajo condiciones de sol y sombra, con humedades relativas entre el 80 y el $90 \%$ y temperaturas de $17-18{ }^{\circ} \mathrm{C}$. La germinación ocurre a partir de la primera semana de siembra sobre un sustrato húmedo (tierra de monte) (Figura 4). La técnica de siembra incluye el entierro de sólo el $50 \%$ de las semillas en el sustrato para facilitar la emergencia de las plántulas. Se ha visto que las

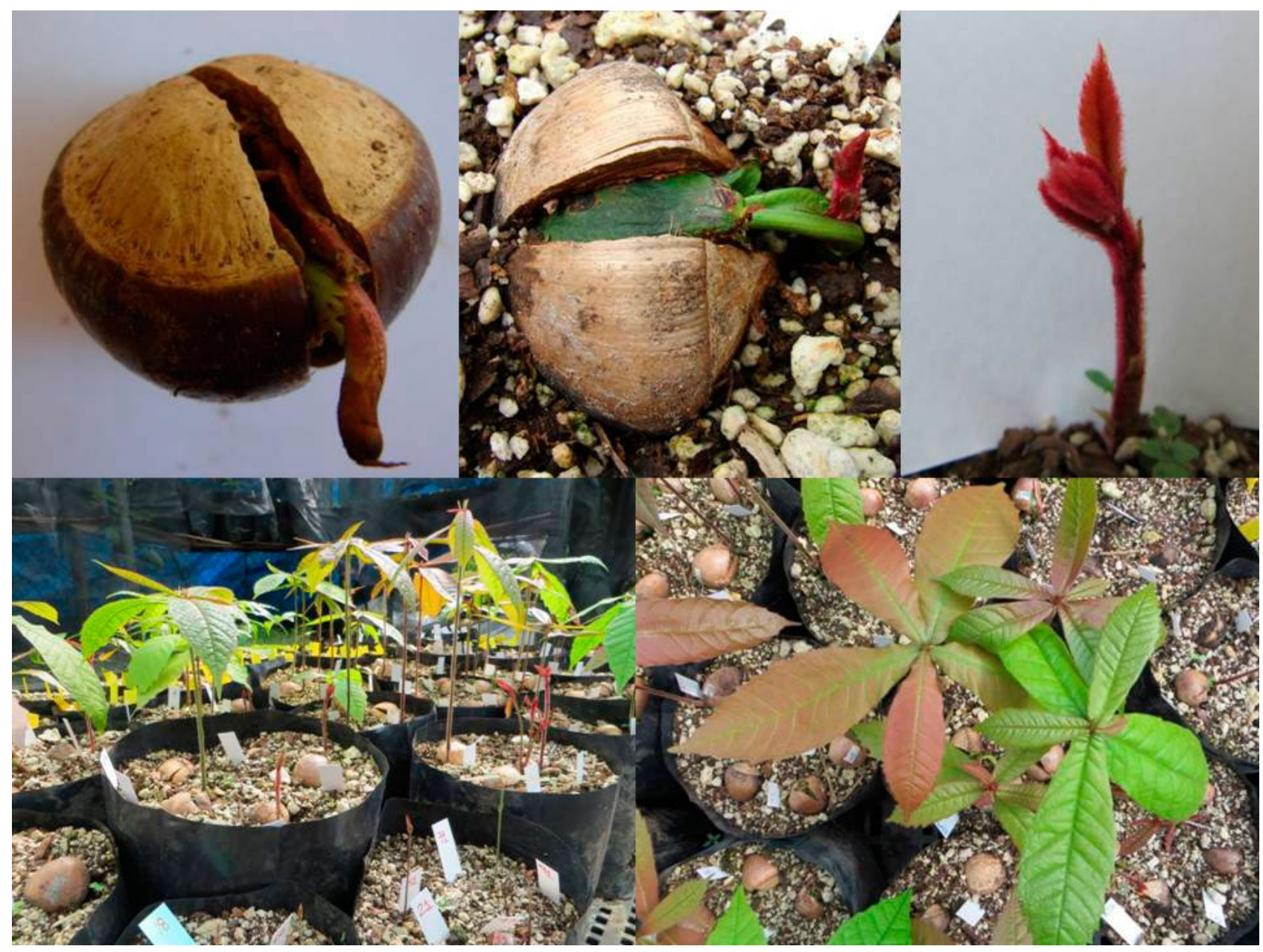

Fig. 4. Germinación y emergencia de plántulas 
bellotas pierden viabilidad dentro de los 60 días a su colecta. Adicionalmente, en este estudio se han registrado alturas de hasta $150 \mathrm{~cm}$ en un año, así como diámetros basales de $2 \mathrm{~cm}$ con una alta producción de follaje, lo que la hace una especie de mediano a rápido crecimiento, estableciéndose con éxito en sitios abiertos o con luz intermedia, es decir, bajo claros creados dentro del bosque.

Durante la etapa de plántula, las hojas pueden ser susceptibles de ataques por hongos o pequeños insectos, para ello, se recomienda emplear un extracto vegetal plaguicida, el cual consiste en emplear 200 a 250 gramos de cola de caballo (Equisetum equisetifolia) o diente de león (Taraxacum officinale) por litro de agua, o bien alcohol, y dejar fermentar de 24 horas a tres días y posteriormente asperjar sobre las hojas de las plantas (Joaquin Becerra-Zavaleta, com. pers.).

De lo anterior, se hace imprescindible el estudio del crecimiento, supervivencia, ecofisiología y anatomía de $Q$. insignis bajo diferentes microambientes para proponer estrategias para restaurar el bosque mesófilo ya que esta especie es de alta importancia forestal, económica y ecológica.

\section{CONCLUSIÓN}

A pesar de que la chicalaba tiene una amplia distribución, sus poblaciones no son muy densas. Además, otros factores perturbadores la colocan en un estado crítico de amenaza en los bosques de niebla en Veracruz, por lo que debe evaluarse con urgencia la posibilidad de incluirla en la Norma Oficial Mexicana-059-SEMARNAT-2010 y facilitar los mecanismos para un manejo y aprovechamiento sustentable por parte de los silvicultores.

Por su parte, en un escenario en el cual el bosque de niebla se ha visto fragmentado y reducido en su superficie a nivel local y regional, es de vital importancia realizar diversos estudios biológicos y ecológicos, que nos permitan conocer su distribución, dinámica poblacional y características ecofisiológicas, indispensables para establecer las bases de programas viables de restauración ecológica. Sin olvidar que, será cada vez más necesario, ayudar a algunas especies vegetales a adaptarse a las actuales condiciones del irreversible cambio climático.

Es deseable la incorporación de diversos enfoques metodológicos que incluyen los estudios anteriormente mencionados que aportarán información valiosa a la ciencia, y además promoverá el entendimiento integral de la bellota más grande del mundo.

\section{REFERENCIAS}

Arizaga, S., Martínez-Cruz, J., Salcedo-Cabrales, M. \& BelloGonzález, M. A. 2009. Manual de la biodiversidad de encinos michoacanos. SEMARNAT, INE. México. 147 pp.

Avendaño-Yáñez, M. L., Ortíz-Ceballos, A. I., SánchezVelásquez, L. R., Pineda-López, M. R. \& Meave, J. A. 2014. Growth of Quercus insignis (Fagaceae) seedling, a native species of the Mexican Cloud Forest. Open Journal of Forestry. 4(1), 1-7.

Axelrod, D. I. 1983. Biogeography of oaks in the ArctoTertiary province. Annals of the Missouri Botanical Garden. 70: 629-657.

Benítez, G., Pulido, Ma. T. \& Equihua, M. 2004. Árboles multiusos nativos de Veracruz para reforestación, restauración y plantaciones. Instituto de Ecología, A.C.; SIGOLFO, CONAFOR. Xalapa, Veracruz, México. 288 pp.

Borgardt, S. J. \& Pigg, K. B. 1999. Anatomical and developmental study of petrified Quercus (Fagaceae) fruits from the middle Miocene, Yakima Canyon, Washington, USA. American Journal of Botany. 86(3): 307-325.

Flora de Nicaragua. 2009. Quercus insignis M. Martens \& Galeotti. Tropicos.org. Missouri Botanical Garden. http://www.tropicos.org/Name/13100166

García-De La Cruz, Y., Ramos Prado, J. M. \& Becerra Zavaleta, J. 2011. Semillas Forestales Nativas para la Restauración Ecológica. CONABIO. Biodiversitas. 94:12-15

González-Espinosa, M., Meave, J. A., Lorea-Hernández, F. G., Ibarra-Maríquez, G. \& Newton, A. 2011. The red list of mexican cloud forest trees. Fauna and Flora International, Botanic Gardens Conservation International, The Global Trees Campaign, The IUCN/SSC Global Tree Specialist Group. Cambridgee, U. K. 149 pp. 
González-Espinosa, M., Meave, J. A., Ramírez-Marcial, N., Toledo-Aceves, T., Lorea-Hernández, F. G. \& IbarraMaríquez, G. 2012. Los bosques de niebla de México: conservación y restauración de su componente arbóreo. Ecosistemas. 21(1-2):36-52.

Guevara, S., Laborde, J. \& Sánchez Ríos, G. 2004. Los Tuxtlas. El paisaje de la sierra. Instituto de Ecología A. C. México. 287 pp.

Kappelle, M. 2006. Neotropical montane oak forests: overview and outlook. In: Kappelle, M. (ed.), Ecological Studies. Ecology and conservation neotropical montane oak forests. Springer-Verlag Berlin Heidelberg. Germany. pp. 449-467.

Kroeber, A. L. 1925. Handbook of the Indians of California. Dover Publication Inc. New York, USA. 995 pp.

Martínez, M. 1981. Los encinos de México. 2da. ed. Anales del Instituto de Biología, México. 358 pp.

Montes-Hernández, B. \& López-Barrera, F. 2013. Seedling establishment of Quercus insignis: A critically endangered oak tree species in southern Mexico. Forest Ecology and Management. 310: 927-934.

Oldfield, S. \& Eastwood, A. 2007. The red list of oaks. Flora and Fauna International. Cambridge, United Kingdom. $32 \mathrm{pp}$.

Real Academia Española. 2001. Diccionario de la Lengua Española. Ed. 22. http://www.rae.es/recursos/diccionarios/drae
Valencia, S. 1995. Contribución al conocimiento del género Quercus (Fagaceae) en el estado de Guerrero, México. Contribuciones del Herbario de la Facultad de Ciencias. Universidad Nacional Autónoma de México. México, D. F. 154 pp.

Valencia, S. 2004. Diversidad del género Quercus (Fagaceae) en México. Boletin de la Sociedad Botánica de México. 75: 33-53.

Williams-Linera, G. 2002. Tree species richness complementarity, disturbance and fragmentation in a Mexican tropical montane cloud forest. Biodiversity and Conservation. 11:1825-1843.

Williams-Linera, G., 2007. El bosque de niebla del centro de Veracruz: ecología, historia y destino en tiempos de fragmentación y cambio climático. Instituto de Ecología, A.C., Veracruz, México. 208 pp.

Williams-Linera, G. 2008. Estructura y diversidad de la vegetación leñosa. En: Manson, R. H., Hernández-Ortiz, V., Gallina, S. \& Mehltreter, K. (eds), Agroecosistemas cafetaleros de Veracruz. Biodiversidad, manejo y conservación. Instituto de Ecología, Instituto Nacional de Ecología, México. pp. 55-68.

Zavala, F. 1989. Identificación de los encinos de México. Universidad Autónoma Chapingo. México. 150 pp. 


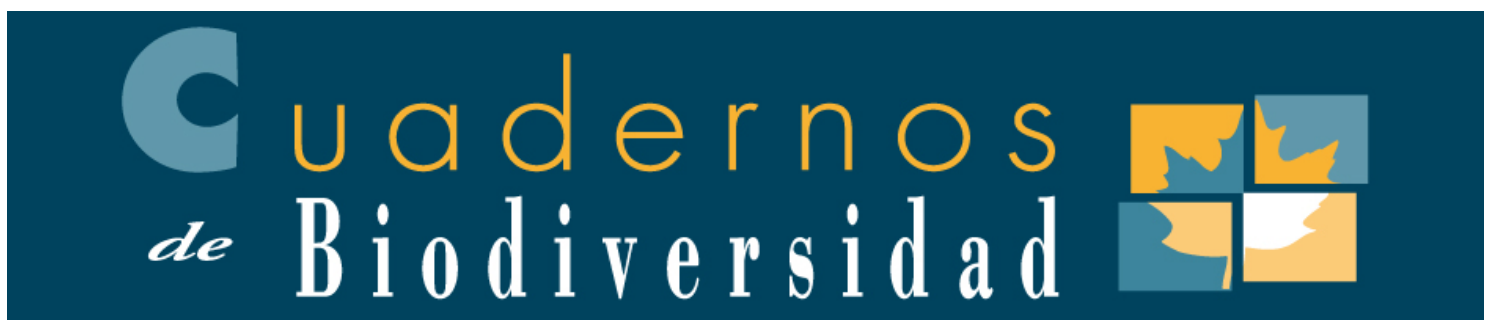

\section{EDITORIAL OFFICE: CIBIO Centro Iberoamericano de la Biodiversidad \\ Universidad de Alicante, E-03080 Alicante (Spain) \\ HOMEPAGE www.cuadernosdebiodiversidad.org}

\section{Fe de erratas}

En la página 1 del artículo "La bellota de Quercus insignis Martens \& Galeotti, 1843, la más grande del mundo", en la parte referida a los autores existe un error en la numeración de los centros de afiliación de los mismos

dice

2 Universidad Autónoma Metropolitana, Unidad Iztapalapa. Laboratorio de Tecnología de la Madera. Av. San Rafael Atlixco 186, Col. Vicentina, C.P. 09340. Delegación Iztapalapa, Distrito Federal, México.

3 Centro de Ecoalfabetización y Diálogo de Saberes, Av. de las Culturas Veracruzanas No. 1, Col. Emiliano Zapata, C.P. 91060. Xalapa, Veracruz, México.

donde debe decir

3 Universidad Autónoma Metropolitana, Unidad Iztapalapa. Laboratorio de Tecnología de la Madera. Av. San Rafael Atlixco 186, Col. Vicentina, C.P. 09340. Delegación Iztapalapa, Distrito Federal, México.

4 Centro de Ecoalfabetización y Diálogo de Saberes, Av. de las Culturas Veracruzanas No. 1, Col. Emiliano Zapata, C.P. 91060. Xalapa, Veracruz, México.

y un error el apellido del segundo autor

dice

J. Becerra-Zalaveta

donde debe decir

J. Becerra-Zavaleta 